\title{
NULL CONTROLLABILITY OF A NONLINEAR HEAT EQUATION
}

\author{
G. ANICULĂESEI AND S. ANIŢA
}

Received 10 January 2002

We study the internal exact null controllability of a nonlinear heat equation with homogeneous Dirichlet boundary condition. The method used combines the Kakutani fixed-point theorem and the Carleman estimates for the backward adjoint linearized system. The result extends to the case of boundary control.

\section{Introduction}

This work is concerned with the internal controllability of the equation

$$
\begin{aligned}
& y_{t}(x, t)-\Delta y(x, t)+a(x, t) y(x, t)+f(t, H y(\cdot, t)) y(x, t) \\
& =m(x) u(x, t), \quad(x, t) \in Q=\Omega \times(0, T), \\
& y(x, t)=0, \quad(x, t) \in \Sigma=\partial \Omega \times(0, T), \\
& y(x, 0)=y_{0}(x), \quad x \in \Omega .
\end{aligned}
$$

Here $\Omega \subset \mathbb{R}^{n}$ is an open, bounded set with a boundary $\partial \Omega, m$ is the characteristic function of a nonempty open subset $\omega$ of $\Omega$, and $\Delta$ is the Laplace operator with respect to the variable $x$.

Here $a: \Omega \times(0, T) \rightarrow \mathbb{R}$ and $f:(0, T) \rightarrow \mathbb{R}$ are given functions satisfying the following conditions:

(i) $a \in L^{\infty}(\Omega \times(0, T))$,

(ii) $f$ is nonnegative and continuous with respect to all variables,

(iii) $f(\cdot, 0) \in L^{\infty}(0, T)$ and $f$ is locally Lipschitz according to the second variable.

Also we assume that

(iv) $H: L^{2}(\Omega) \rightarrow \mathbb{R}$ is a locally Lipschitz continuous operator and $y_{0} \in L^{2}(\Omega)$. Equation (1.1) describes the heat propagation with a viscosity term. 
System (1.1) is said to be null controllable if for every $T>0$ there are $(y, u) \in$ $C\left([0, T] ; L^{2}(\Omega)\right) \cap W_{\text {loc }}^{1,2}\left((0, T] ; L^{2}(\Omega)\right) \cap L^{2}\left(0, T ; H_{0}^{1}(\Omega)\right) \cap L_{\mathrm{loc}}^{2}\left((0, T] ; H^{2}(\Omega)\right) \times$ $L^{2}(Q)$, which satisfy (1.1) and such that $y(x, T)=0$ a.e. $x \in \Omega$.

The main result of the paper amounts to saying that system (1.1) is null controllable for all $y_{0} \in L^{2}(\Omega)$.

Null controllability of the linear heat equation, when the control acts on a subset of the domain $\Omega$, was established by Lebeau and Robbiano [6] and was extended later by Fursikov and Imanuvilov [5] to the semilinear equation,

$$
y_{t}(x, t)-\Delta y(x, t)+f(y(x, t))=m(x) u(x, t), \quad(x, t) \in Q
$$

where $f$ is a sublinear function.

Fernández-Cara [4] established null controllability of superlinear control system of the form

$$
y_{t}(x, t)-\Delta y(x, t)+f(y(x, t)) y(x, t)=m(x) u(x, t), \quad(x, t) \in Q,
$$

with $f$ satisfying the condition $f(y)(\log |y|+1)^{-1} \rightarrow 0$ as $|y| \rightarrow \infty$ while Barbu [3] established the same result in the case $f(y)(\log |y|+1)^{-3 / 2} \rightarrow 0$ as $|y| \rightarrow \infty$ if $1 \leq n<6$.

A general discussion on dissipative semilinear heat equation has been done by Aniţa and Tataru [1]. It has been proved that if $f$ is nonnegative and is growing at infinity faster than a polynomial, then the equation is not null controllable.

This is not the case of our problem. Here, we show that system (1.1) is null controllable for any $f$ satisfying the hypotheses mentioned above. Anyway, in (1.1) the nonlinear term $f(t, H y(\cdot, t))$ does not depend explicitly on the spatial variable.

The paper is organized as follows. The main result is stated in Section 2 and proved in Section 3 via the Kakutani fixed-point theorem. The proof is based on Carleman inequality for the backward adjoint linearized system associated with (1.1). We do not impose asymptotic conditions on $f$ (as in $[3,4]$ ).

In what follows we use standard notations for the Sobolev spaces $H^{2}(\Omega)$, $H_{0}^{1}(\Omega)$, and $L^{2}(\Omega)$ on $\Omega$ and $Q$. Denote by $|\cdot|$ the usual norm of $\mathbb{R}^{n}$, and by $(\cdot, \cdot)$ the inner product of $L^{2}(\Omega)$. Moreover, we set

$$
\begin{aligned}
& W^{1,2}\left(0, T ; L^{2}(\Omega)\right)=\left\{y \in L^{2}\left(0, T ; L^{2}(\Omega)\right) ; \frac{d y}{d t} \in L^{2}\left(0, T ; L^{2}(\Omega)\right)\right\} \\
& W_{\text {loc }}^{1,2}\left(0, T ; L^{2}(\Omega)\right)=\cap_{\delta \in(0, T)} W^{1,2}\left(\delta, T ; L^{2}(\Omega)\right)
\end{aligned}
$$

where $d y / d t$ is taken in the sense of distributions. 


\section{The main result}

Theorem 2.1. Assume that conditions (i), (ii), (iii), and (iv) hold. Then for all $y_{0} \in L^{2}(\Omega)$ and $T>0$, there are $u \in L^{2}(Q)$ and $y \in C\left([0, T] ; L^{2}(\Omega)\right) \cap$ $W_{\text {loc }}^{1,2}\left((0, T] ; L^{2}(\Omega)\right) \cap L^{2}\left(0, T ; H_{0}^{1}(\Omega)\right) \cap L_{\text {loc }}^{2}\left((0, T] ; H^{2}(\Omega)\right)$, which satisfy (1.1), and

$$
y(x, T)=0 \quad \text { a.e. } x \in \Omega \text {. }
$$

The result of Theorem 2.1 extends in a classical manner (see [3]) to the case of boundary control. More exactly we have the following result.

Theorem 2.2. Under assumptions (i), (ii), (iii), and (iv) for each $T>0$ and $y_{0} \in$ $H^{1}(\Omega)$, there are $v \in L^{2}(\Sigma)$ and $y \in W^{1,2}\left([0, T] ; L^{2}(\Omega)\right) \cap L^{2}\left(0, T ; H^{2}(\Omega)\right)$, which satisfy

$$
\begin{gathered}
y_{t}-\Delta y+a(x, t) y+f(t, H y) y=0 \quad \text { in } Q, \\
y=v \quad \text { on } \Sigma, \\
y(x, 0)=y_{0} \quad \text { in } \Omega, \\
y(x, T)=0 \quad \text { in } \Omega .
\end{gathered}
$$

Proof of Theorem 2.2. Let $\tilde{\Omega}$ be an open bounded set such that $\tilde{\Omega} \supset \Omega$. We set $\omega=\tilde{\Omega} \backslash \bar{\Omega}$ and apply Theorem 2.1 with $y_{0} \in H^{1}(\Omega)$ to $(1.1)$ on $\tilde{\Omega}$ with Dirichlet boundary condition, and the initial value condition $y(x, 0)=\tilde{y}_{0}(x)$ on $\tilde{\Omega}$ where $\tilde{y}_{0}$ is an $H_{0}^{1}$-extension of $y_{0}$ to $\tilde{\Omega}$.

Consequently, there is $\tilde{y}$ satisfying $(1.1)$ on $\tilde{\Omega} \times(0, T)$ such that $\tilde{y}(T)=0$. So, by the trace theorem $v=\tilde{y}$ on $\partial \Omega \times(0, T)$ belongs to $L^{2}(\Sigma)$ and $y$, the restriction of $\tilde{y}$ to $\Omega \times(0, T)$ satisfies the requirements of Theorem 2.2 .

\section{Proof of Theorem 2.1}

Firstly, we prove Theorem 2.1 in the case $y_{0} \in H_{0}^{1}(\Omega)$.

We fix $y_{0} \in H_{0}^{1}(\Omega)$ and define the set

$$
K=\left\{w \in L^{\infty}\left(0, T ; L^{2}(\Omega)\right) ;\|w(t)\|_{L^{2}(\Omega)} \leq M \text {, a.e. } t \in(0, T)\right\},
$$

where $M$ is a positive constant to be defined later.

For $w \in K$ and $\mu \in L^{2}(Q)$ consider the linear system

$$
\begin{gathered}
y_{t}-\Delta y+a(x, t) y+f(t, H w(t)) y=\mu \quad \text { in } Q, \\
y=0 \quad \text { on } \Sigma, \\
y(x, 0)=y_{0} \quad \text { in } \Omega .
\end{gathered}
$$


We note first that for all $w \in K, u \in L^{2}(Q)$, and $y_{0} \in H_{0}^{1}(\Omega)$, (3.2) has a unique solution

$$
y=y^{u} \in L^{2}\left(0, T ; H_{0}^{1}(\Omega) \cap H^{2}(\Omega)\right) \cap W^{1,2}\left(0, T ; L^{2}(\Omega)\right) .
$$

We give a sketch of the proof for this assertion. Since $H: L^{2}(\Omega) \rightarrow \mathbb{R}$ is locally Lipschitz continuous, for $w \in L^{\infty}\left(0, T ; L^{2}(\Omega)\right)$ it follows that $H w \in L^{\infty}(0, T)$.

Now, assumptions (ii) and (iii) imply that $f(\cdot, H w(\cdot)) \in L^{\infty}(0, T)$ for all $w \in K$.

Along with (i), the last implies that $\tilde{a} \in L^{\infty}(Q)$ where $\tilde{a}(x, t)=-a(x, t)-$ $f(t, H w(t))$, for all $w \in K$.

Let $S(t)$ be the $C_{0}$-semigroup generated on $L^{2}(\Omega)$ by the Laplace operator with Dirichlet boundary value conditions. Then, the solution $y$ to (3.2) (if it exists) can be represented by the variation of constant formula,

$$
y(t)=S(t) y_{0}+\int_{0}^{t} S(t-s)(\tilde{a}(s) y(s)+\mu(s)) d s .
$$

In a standard way (see [2]) we show that (3.4) has a unique solution, $y \in$ $C\left([0, T] ; L^{2}(\Omega)\right)$, provided that the operator $\mathscr{T}: C\left([0, T] ; L^{2}(\Omega)\right) \rightarrow C([0, T]$; $\left.L^{2}(\Omega)\right)$,

$$
(\mathscr{T} y)(t)=\int_{0}^{t} S(t-s)(\tilde{a}(s) y(s)+\mu(s)) d s
$$

is a contraction.

Multiplying now (3.2) by $y$ and integrating on $(0, t) \times \Omega$, we obtain

$$
\|y(t)\|_{L^{2}(\Omega)}^{2} \leq A+B \int_{0}^{t}\|y(s)\|_{L^{2}(\Omega)}^{2} d s
$$

where $A$ and $B$ are positive constants. Then, Gronwall's inequality gives

$$
\|y(t)\|_{L^{2}(\Omega)} \leq \bar{C} \quad \forall t \in[0, T],
$$

$\bar{C}$ being a positive constant (independent of $w \in K$ ).

As $\tilde{a} \in L^{\infty}(Q), y \in L^{2}(Q)$, and $u \in L^{2}(Q)$, it follows that $\tilde{a} y+\mu \in L^{2}(Q)$ and by [2, Theorem 2.1, page 189] we conclude that the solution $y$ of (3.2) satisfies (3.3).

Multiplying now (3.2) by $y_{t}-\Delta y$ and having in mind (3.7), the following inequality is obtained

$$
\begin{aligned}
&\|y(t)\|_{H_{0}^{1}(\Omega)}^{2}+\int_{Q}\left(y_{t}^{2}(x, t)+|\Delta y(x, t)|^{2}\right) d x d t \\
& \leq \tilde{\mu}\left(M,\left\|y_{0}\right\|_{H_{0}^{1}(\Omega)}\right)+\int_{Q} \mu^{2} d x d t,
\end{aligned}
$$

where $\tilde{\mu}$ is a constant depending on $M$ and $y_{0}$. 
Now consider the optimal control problem $(\varepsilon>0)$,

$$
\text { Minimize }\left\{\frac{1}{\varepsilon} \int_{\Omega} y^{2}(x, T) d x+\int_{Q} u^{2} d x d t\right\}
$$

subject to (3.2).

It is easy to observe that (in (3.2)) the map $u \rightarrow y^{u}$ is closed in $\left(L^{2}(Q)\right)_{w} \times$ $L^{2}(Q)$, where by $\left(L^{2}(Q)\right)_{w}$ we have denoted the space $L^{2}(Q)$ endowed with the weak topology. This implies that there exists an optimal pair $\left(y_{\varepsilon}, u_{\varepsilon}\right)$ for the functional (3.9).

The Pontryagin maximum principle yields that

$$
u_{\varepsilon}(x, t)=m(x) p_{\varepsilon}(x, t) \quad \text { a.e. }(x, t) \in Q
$$

where $p_{\varepsilon}$ is the solution to the backward adjoint system

$$
\begin{gathered}
\left(p_{\varepsilon}\right)_{t}+\Delta p_{\varepsilon}-a p_{\varepsilon}-f(t, H w(t)) p_{\varepsilon}=0 \quad \text { in } Q, \\
p_{\varepsilon}=0 \quad \text { on } \Sigma, \\
p_{\varepsilon}(x, T)=-\frac{1}{\varepsilon} y_{\varepsilon}(x, T) \quad \text { in } \Omega .
\end{gathered}
$$

Now, we prove an observability result for the solution $p \in L^{2}\left(0, T ; H_{0}^{1}(\Omega)\right) \cap$ $C\left([0, T] ; L^{2}(\Omega)\right)$ to the equation

$$
p_{t}+\Delta p-a(x, t) p-f(t, H w(t)) p=0 \quad \text { in } Q .
$$

Lemma 3.1. There is a constant $C$ independent of $w, M$, and $p$ such that

$$
\int_{\Omega} p^{2}(x, 0) d x \leq C \int_{0}^{T} \int_{\omega} p^{2}(x, t) d x d t
$$

Proof. Consider the problem

$$
\begin{gathered}
p_{t}+\Delta p-a p=0 \quad \text { in } Q, \\
p=0 \quad \text { on } \Sigma, \\
p(x, T)=z(x) \quad \text { in } \Omega,
\end{gathered}
$$

where $z \in L^{2}(\Omega)$.

It is well known (see [5]) that the solution of (3.14) satisfies the Carleman inequality,

$$
\int_{\Omega} p^{2}(x, 0) d x \leq C \int_{0}^{T} \int_{\omega} p^{2}(x, t) d x d t
$$

for all $z \in L^{2}(\Omega)$. 
It is easy to observe that the solution to (3.12) is given by

$$
p_{\varepsilon}(t)=e^{-\int_{t}^{T} f(s, H w(s)) d s} p(t)
$$

which implies that

$$
\begin{gathered}
\int_{\Omega} p_{\varepsilon}^{2}(x, 0) d x=e^{-2 \int_{0}^{T} f(s, H w(s)) d s} \int_{\Omega} p^{2}(x, 0) d x \\
\int_{0}^{T} \int_{\Omega} p_{\varepsilon}^{2}(x, t) d x d t=\int_{0}^{T}\left(e^{-2 \int_{t}^{T} f(s, H w(s)) d s} \int_{\Omega} p^{2}(x, t) d x\right) d t .
\end{gathered}
$$

Now inequality (3.15) and $f \geq 0$ imply that

$$
\begin{aligned}
e^{-2 \int_{0}^{T} f(s, H w(s)) d s} \int_{\Omega} p^{2}(x, 0) d x & \leq C e^{-2 \int_{0}^{T} f(s, H w(s)) d s} \int_{0}^{T} \int_{\omega} p^{2}(x, t) d x d t \\
& \leq C \int_{0}^{T} e^{-2 \int_{t}^{T} f(s, H w(s)) d s} \int_{\omega} p^{2}(x, t) d x d t \\
& =C \int_{0}^{T} \int_{\omega} p_{\varepsilon}^{2}(x, t) d x d t
\end{aligned}
$$

and thus $p_{\varepsilon}$ verifies (3.13) ending the proof of the lemma.

Remark 3.2. The result given by the lemma can be viewed as a uniform observability result for the linear adjoint system (3.11) with respect to $w \in K$.

Proof of Theorem 2.1 (continued). Multiplying (3.2) by $p_{\varepsilon},(3.11)$ by $y_{\varepsilon}$, and having in mind (3.10), we obtain, after integration on $Q$ that

$$
\begin{aligned}
\frac{1}{\varepsilon} \int_{\Omega} y_{\varepsilon}^{2}(x, T) & d x+\int_{0}^{T} \int_{\omega} u_{\varepsilon}^{2}(x, t) d x d t \\
& =-\int_{\omega} y_{\varepsilon}(x, 0) p_{\varepsilon}(x, 0) d x \\
& =-\int_{\Omega} y_{0}(x) p_{\varepsilon}(x, 0) d x \\
& \leq \gamma \int_{\Omega} p_{\varepsilon}^{2}(x, 0)+\frac{1}{\gamma} \int_{\Omega} y_{0}^{2}(x) d x \quad \forall \gamma>0 .
\end{aligned}
$$

As $p_{\varepsilon}$ satisfies (3.13), the latter implies that

$$
\begin{aligned}
& \frac{1}{\varepsilon} \int_{\Omega} y_{\varepsilon}^{2}(x, T) d x+\int_{0}^{T} \int_{\omega} u_{\varepsilon}^{2}(x, t) d x d t \\
& \leq C \gamma \int_{0}^{T} \int_{\omega} u_{\varepsilon}^{2}(x, t) d x d t+\frac{1}{\gamma} \int_{\Omega} y_{0}^{2}(x) d x \quad \forall \gamma>0
\end{aligned}
$$


which gives

$$
\frac{1}{\varepsilon} \int_{\Omega} y_{\varepsilon}^{2}(x, T) d x \leq C_{1}, \quad \int_{0}^{T} \int_{\omega} u_{\varepsilon}^{2}(x, t) d x d t \leq C_{1},
$$

$C_{1}$ being a positive constant.

By estimates (3.21) it follows that, selecting a subsequence, we have

$$
\begin{array}{ll}
u_{\varepsilon} \longrightarrow u & \text { weakly in } L^{2}(Q), \\
y_{\varepsilon} \longrightarrow y & \text { weakly in } L^{2}\left(0, T ; H_{0}^{1}(\Omega) \cap H^{2}(\Omega)\right) \cap W^{1,2}\left(0, T ; L^{2}(\Omega)\right),
\end{array}
$$

where $(y, u)$ satisfies $(3.2)$ and $y(T) \equiv 0$.

For each $w \in K$, we denote by $\Phi(w) \subset L^{2}(Q)$ the set of all solutions $y^{u} \in$ $L^{2}\left(0, T ; H_{0}^{1}(\Omega) \cap H^{2}(\Omega)\right) \cap W^{1,2}\left(0, T ; L^{2}(\Omega)\right)$ to (3.2) such that

$$
y^{u}(T)=0, \quad\|u\|_{L^{2}(Q)} \leq C_{1}^{1 / 2} .
$$

By (3.21) and (3.22) we deduce that $\Phi(w) \neq \varnothing$ for each $w \in K$. Moreover, it is readily seen that $\Phi(w)$ is a convex subset of $L^{2}(Q)$. Since, by (3.8)

$$
u_{n} \longrightarrow u \quad \text { weakly in } L^{2}(Q)
$$

implies that

$$
y^{u_{n}} \longrightarrow y \quad \text { in } L^{2}(Q)
$$

it follows also that $\Phi(w)$ is a closed subset of $L^{2}(Q)$. At the same time from estimate (3.8) we deduce, via the Arzelà-Ascoli theorem that $\Phi(K)$ is relatively compact.

Multiplying once again (3.2) by $y$ and integrating on $Q_{t}=\Omega \times(0, t)$, we obtain

$$
\|y(t)\|_{L^{2}(\Omega)} \leq M \quad \forall t \in(0, T)
$$

which is a constant that we choose in the definition of $K$. So, we have proved that $\Phi(K) \subset K$. Finally, we prove that $\Phi$ is upper semicontinuous in $L^{2}(Q) \times L^{2}(Q)$. For this, let $w_{n} \in K, y_{n} \in \Phi\left(w_{n}\right), y_{n}=y^{u_{n}}$ such that

$$
\begin{array}{ll}
w_{n} \longrightarrow w & \text { in } L^{2}(Q), \\
y_{n} \longrightarrow y & \text { in } L^{2}(Q) .
\end{array}
$$

By estimate (3.8) it follows that, eventually on a subsequence,

$$
\begin{array}{ll}
u_{n} \longrightarrow u & \text { weakly in } L^{2}(Q), \\
y_{n} \longrightarrow y & \text { strongly in } C\left([0, T] ; L^{2}(\Omega)\right) \text { and } \\
& \text { weakly in } L^{2}\left(0, T ; H_{0}^{1}(\Omega) \cap H^{2}(\Omega)\right) \cap W^{1,2}\left(0, T ; L^{2}(\Omega)\right) .
\end{array}
$$


382 Null controllability of a nonlinear heat equation

So, we have

$$
\begin{gathered}
f\left(t, H w_{n}(t)\right) y_{n}(x, t) \longrightarrow f(t, H w(t)) y(x, t) \quad \text { a.e. in } Q, \\
f\left(t, H w_{n}\right) y_{n} \longrightarrow \eta \quad \text { weakly in } L^{2}(Q) .
\end{gathered}
$$

By Egorov's theorem we conclude that

$$
\eta=f(t, H w(t)) y(x, t) \quad \text { a.e. in } Q \text {. }
$$

Since $y_{n}$ is a solution of

$$
\begin{gathered}
\left(y_{n}\right)_{t}-\Delta y_{n}+a(x, t) y_{n}+f\left(t, H w_{n}\right) y_{n}=\mu_{n} \quad \text { in } Q, \\
y_{n}=0 \quad \text { on } \Sigma, \\
y_{n}(x, 0)=y_{0}(x), \quad y_{n}(x, T)=0 \quad \text { in } \Omega,
\end{gathered}
$$

we get (by passing to the limit) that $(y, u)$ satisfies $(3.2)$ and $(3.23)$, that is, $y \in \Phi(w)$ as claimed. By the Kakutani fixed-point theorem in $L^{2}(Q)$ satisfied by $\Phi$, we infer that there is at least one $w \in K$ such that $w \in \Phi(w)$. Then, by the definition of $\Phi$, this implies that there exists at least one pair $(y, u)$ satisfying the conditions of Theorem 2.1.

In the general case $y_{0} \in L^{2}(\Omega)$, we can use the smoothing effect of the parabolic equation on the initial data. More exactly, for each $\varepsilon>0$ there exists $\tilde{\varepsilon} \in$ $(0, \varepsilon]$ such that $\bar{y}(\tilde{\varepsilon}) \in H_{0}^{1}(\Omega), \bar{y}$ being the solution of $(1.1)$ with $u \equiv 0$ on $\omega \times$ $(0, \varepsilon)$ (see $[2])$.

Theorem 2.1 applies, for example, to the semilinear heat equation with a viscosity term,

$$
\begin{gathered}
y_{t}-\Delta y+a y+f\left(t, \int_{\Omega} y(x, t) d x\right) y=\mu \text { in } Q, \\
y=0 \quad \text { on } \Sigma, \\
y(x, 0)=y_{0}(x) \text { in } \Omega .
\end{gathered}
$$

Here $a$ and $f$ satisfy conditions (i), (ii), and (iii).

\section{References}

[1] S. Anița and D. Tataru, Null controllability of the dissipative semilinear heat equation, submitted to SIAM J. Control Optimiz.

[2] V. Barbu, Nonlinear Semigroups and Differential Equations in Banach Spaces, Editura Academiei Republicii Socialiste România, Bucharest, 1976.

[3] Exact controllability of the superlinear heat equation, Appl. Math. Optim. 42 (2000), no. 1, 73-89.

[4] E. Fernández-Cara, Null controllability of the semilinear heat equation, ESAIM Control Optim. Calc. Var. 2 (1997), 87-103. 
[5] A. V. Fursikov and O. Yu. Imanuvilov, Controllability of Evolution Equations, Lecture Notes Series, vol. 34, Seoul National University, Research Institute of Mathematics, Global Analysis Research Center, Seoul, 1996.

[6] G. Lebeau and L. Robbiano, Contrôle exact de l'équation de la chaleur, Comm. Partial Differential Equations 20 (1995), no. 1-2, 335-356 (French).

G. Aniculăesei: Department of Mathematics, "Alexandru Ioan Cuza” University of Iaşı, 11 Carol I Boulevard, Iaşi 6600, Romania

E-mail address: gani@uaic.ro

S. Aniţa: Department of Mathematics, "Alexandru Ioan Cuza" University and Institute of Romanian Academy, Iaşi 6600, Romania

E-mail address: sanita@uaic.ro 


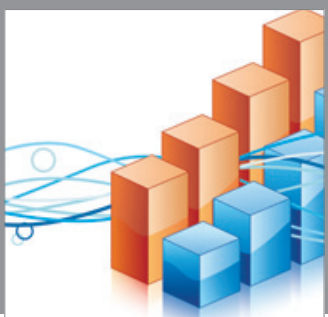

Advances in

Operations Research

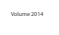

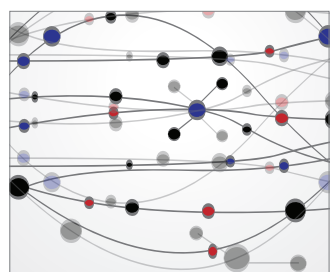

\section{The Scientific} World Journal
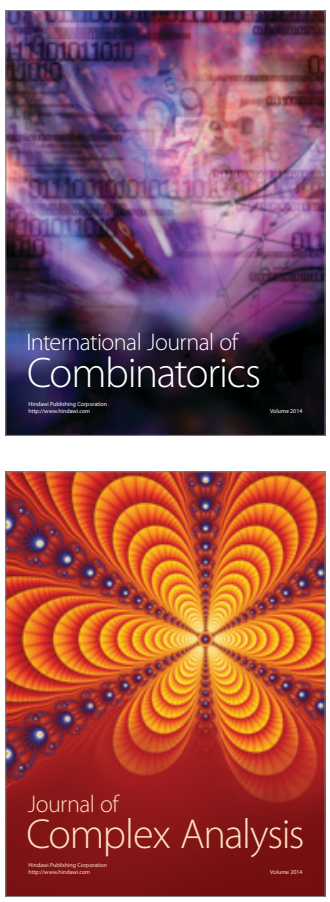

International Journal of

Mathematics and

Mathematical

Sciences
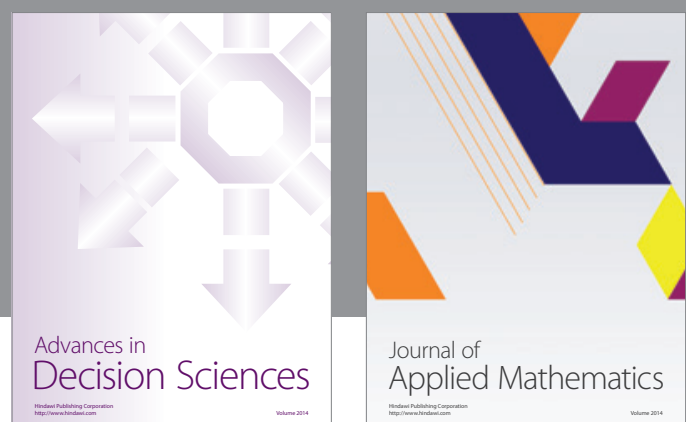

Journal of

Applied Mathematics
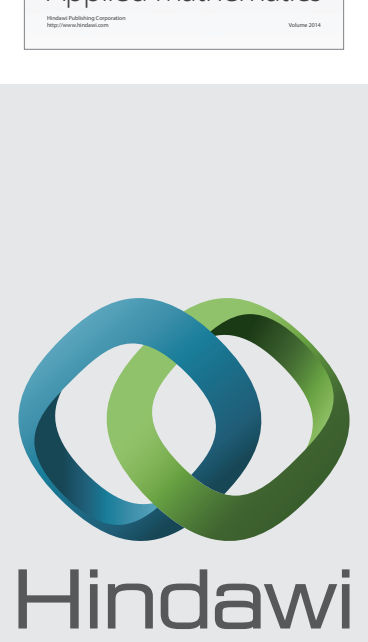

Submit your manuscripts at http://www.hindawi.com
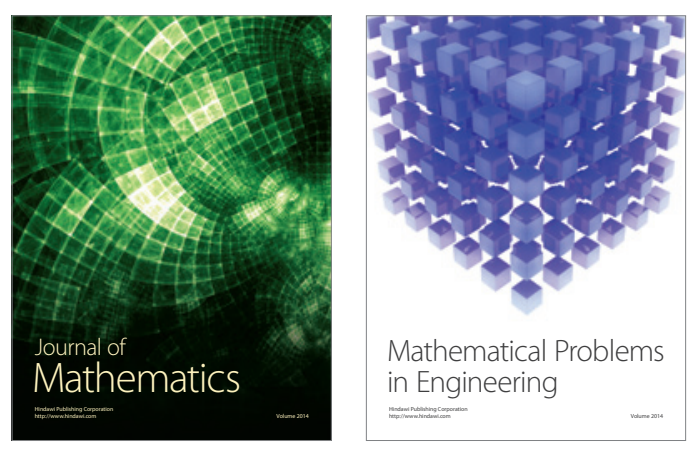

Mathematical Problems in Engineering
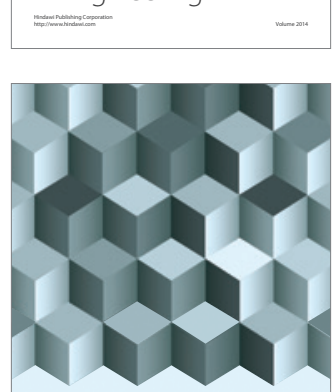

Journal of

Function Spaces
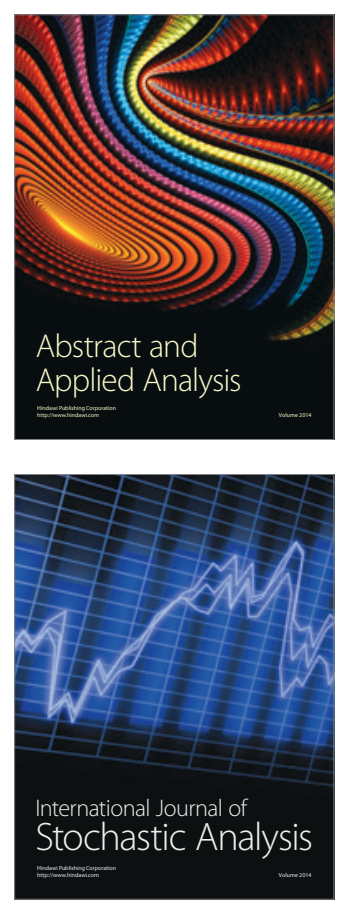

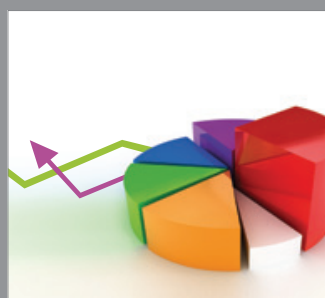

ournal of

Probability and Statistics

Promensencen
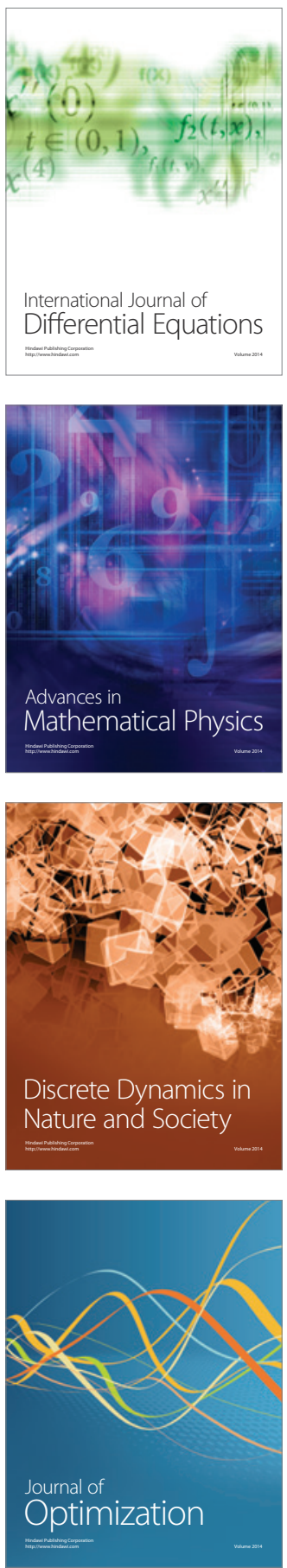